\title{
Pediatric neurotoxocariasis with concomitant cerebral, cerebellar, and peripheral nervous system involvement: case report and review of the literature
}

\author{
Sócrates Salvador, ${ }^{1}$ Ricardo Ribeiro, ${ }^{1}$ Maria Isabel Winckler, ${ }^{2}$ Lygia Ohlweiler, $^{2}$ Rudimar Riesgo $^{2}$
}

\begin{abstract}
Objective: To alert pediatricians to the neurologic consequences of toxocariasis and to describe the first pediatric case of neurotoxocariasis with concomitant cerebral, cerebellar and peripheral nervous system involvement.

Description: We report a case of neurotoxocariasis in a previously healthy 5-year-old boy with unusual symptoms and multi-site involvement of both the central and peripheral nervous system. Differential diagnoses are discussed and the relevant literature is reviewed. Since the early 1950s, fewer than fifty cases have been described, mostly in adult patients.

Comments: Although human toxocariasis is one of the most common zoonotic helminth infections, neurotoxocariasis is a rare condition, especially in pediatric patients. Although toxocariasis usually presents as a self-limiting disease with no central nervous system involvement, when it does occur, it can be devastating. Neurotoxocariasis should be added to the differential diagnosis of pediatric patients with unusual neurologic symptoms accompanied by high levels of eosinophils in the cerebrospinal fluid. Early diagnosis and treatment can prevent long-term neurologic sequelae.
\end{abstract}

J Pediatr (Rio J). 2010;86(6):531-534: Pediatrics, nervous system, toxocariasis.

\section{Introduction}

Parasitic infections, some of which may affect the central nervous system (CNS), are a major public health concern. ${ }^{1}$ The worldwide prevalence of helminthic infestations remains high, despite significant progress on basic sanitation and increased access to anthelmintic treatment.2,3

Toxocariasis, transmitted to humans by the ingestion of Toxocara canis (or, occasionally, Toxocara cati) eggs, is one of the most common helminthiases. The seroprevalence of
Toxocara canis in Brazil is estimated to be approximately $39 \%$ in children between six months and six years of age. There are three main clinical presentations of toxocariasis; neurotoxocariasis is included in the systemic form of the disease, known as visceral larva migrans. 3,4

Neurotoxocariasis is a rare condition. Accordingly, fewer than twenty-five cases have been described in the pediatric age group since 1951.3 Toxocara infection of

1. MD. Department of Pediatrics, Child Neurology Unit, Hospital de Clínicas de Porto Alegre (HCPA), Universidade Federal do Rio Grande do Sul (UFRGS), Porto Alegre, RS, Brazil.

2. MD, PhD. Department of Pediatrics, Child Neurology Unit, HCPA, UFRGS, Porto Alegre, RS, Brazil.

No conflicts of interest declared concerning the publication of this article.

Suggested citation: Salvador S, Ribeiro R, Winckler MI, Ohlweiler L, Riesgo R. Pediatric neurotoxocariasis with concomitant cerebral, cerebellar, and peripheral nervous system involvement: case report and review of the literature. J Pediatr (Rio J). 2010;86(6):531-534

Manuscript submitted Jun 07 2010, accepted for publication Aug 302010

doi:10.2223/JPED.2037 
the CNS usually presents as one or more of the following neurologic conditions: eosinophilic meningitis, ${ }^{5}$ encephalitis/ meningoencephalitis; encephalitis associated with vasculitis ${ }^{6}$; arachnoiditis and spinal cord lesions ${ }^{7}$; or even epilepsy. ${ }^{8}$ Larval migration to the CNS is exceedingly rare. Tissue damage is caused by inflammatory reaction rather than by the infestation itself: the larvae produce glycosylated proteins that induce a CD4-Th2 response, with production of IgE and interleukin-5, which ultimately promotes vascular adhesion and eosinophilic differentiation. ${ }^{6}$

We present a rare clinical presentation of acute neurotoxocariasis in a pediatric patient with unusual concomitant symptoms, affecting both the CNS and the peripheral nervous system (PNS), as well as the musculoskeletal system. No similar cases were found in a LILACS and MEDLINE/PubMed database search.

Informed consent authorizing the publication of this case report was provided by the patient's parents.

\section{Case report}

A previously healthy 5-year-old male, living in a poor neighborhood without appropriate access to hygiene and sanitation, presented with neurologic complaints of two days' duration. The patient reported prostration, drowsiness, and myalgia, but no seizures. Initial neurologic assessment revealed limited range of motion, probably secondary to myalgia. Gait and balance difficulties were also noted. The initial clinical hypotheses of myositis or poisoning were ruled out by laboratory testing. On the next day, ataxia became more prominent, with impairments in gait, balance, and motor coordination, in addition to dysmetria. Simultaneously, the patient began to show meningeal signs. Later on the same day, cognitive impairment, mental confusion, and altered level of consciousness were observed; the patient also developed urinary and fecal incontinence.

Head CT showed subtle changes in the density of the cerebellar region, but no unequivocal lesions, cysts, or larvae were visible. Cerebrospinal fluid (CSF) tests showed increased cell counts and eosinophilia, with eosinophils comprising $27 \%$ of CSF leukocytes. A complete blood count also revealed leukocytosis with $35 \%$ eosinophils. Due to the high likelihood of eosinophilic meningoencephalitis, serological testing for Toxocara canis was performed on blood and CSF samples. Empirical anthelmintic treatment with thiabendazole $50 \mathrm{mg} / \mathrm{kg}$ twice daily was immediately started. In the meantime, serological tests came back positive for Toxocara canis, with high titres in both samples. Stool ova and parasites exam was negative for eggs or larvae.

On the third day of treatment, the patient regained bladder and bowel control, and subsequently began to show improvement in limb motor coordination and balance. On the 15th day of treatment, he was able to sit unaided.

Electroencephalography (EEG) revealed signs of diffuse cerebral involvement, characterized by moderate multifocal epileptiform activity (Figure 1). Brain and cerebellar magnetic resonance imaging (MRI) were normal, whereas spine MRI showed mild contrast enhancement in the lower sacral nerve roots (Figure 2). Electromyography showed changes consistent with polyneuropathy.

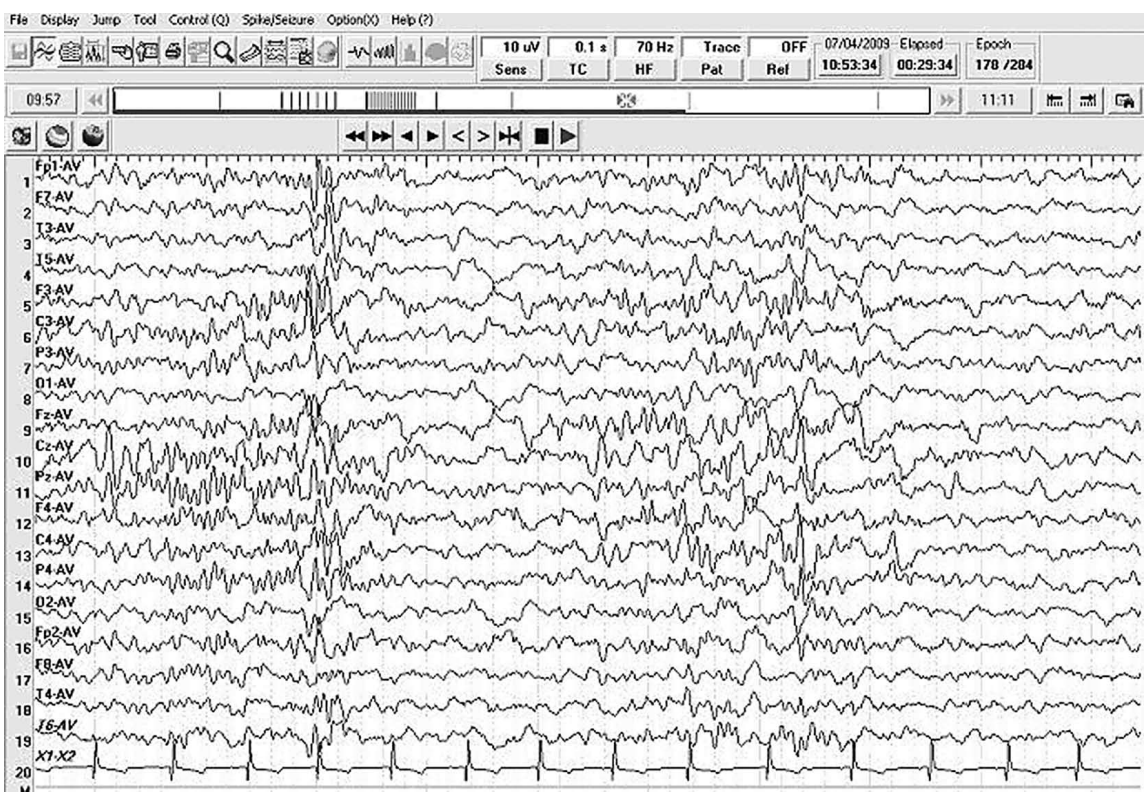

Figure 1 - Abnormal sleep electroencephalogram, with moderate multifocal epileptiform discharges 


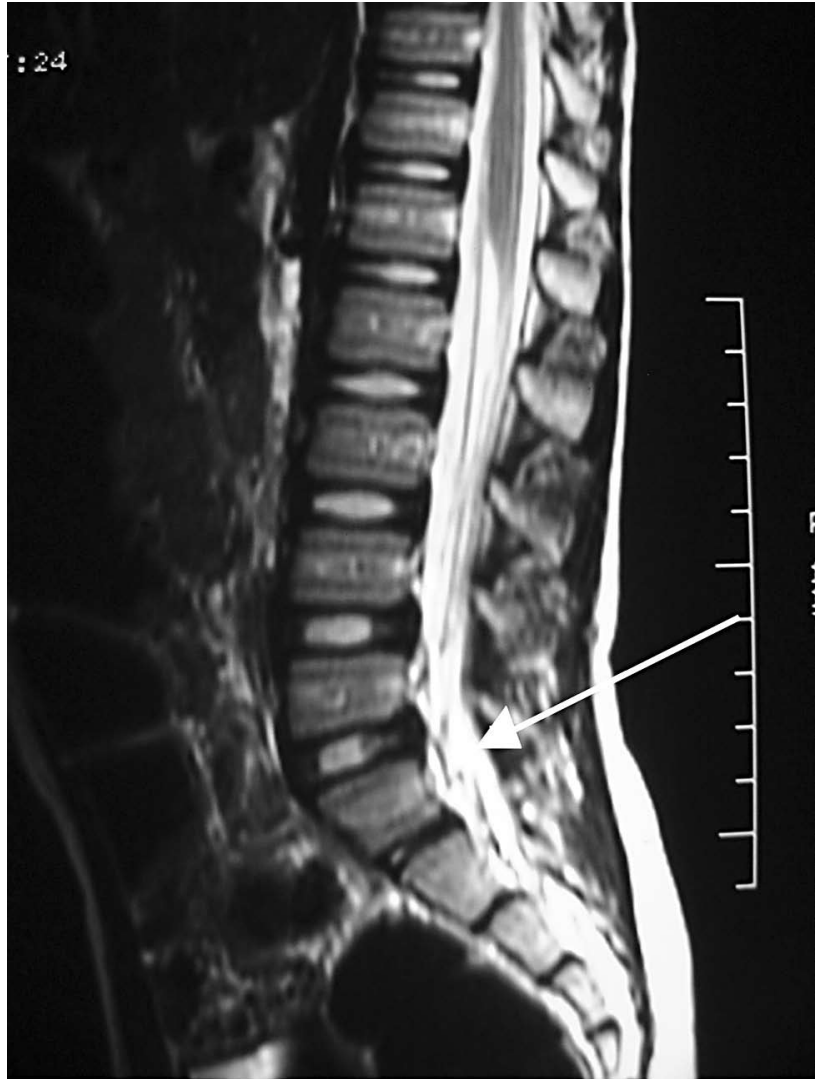

Figure 2 - Spinal MRI showing slight enhancement of the lower sacral nerve roots

By the 25th day of treatment, the patient had improved markedly, and he was discharged from hospital approximately one month after admission, with mild gait impairment still present. Five additional weeks of thiabendazole treatment were prescribed, along with a functional rehabilitation plan. Two months after discharge, the patient's neurological examination was completely normal.

\section{Discussion}

The nematodes (Nematoda) are the second largest phylum in the animal kingdom. Globalization has increased the risk of parasitic infection; toxocariasis is now a worldwide health concern, ${ }^{8}$ and is endemic in several parts of the world. ${ }^{2}$ Among the nematodes, Toxocara is one of the five genera that most often affect the CNS. Although toxocariasis usually presents as a self-limiting disease with no neurologic involvement, when CNS infection does occur, it can be devastating. ${ }^{1,2}$

Our patient was previously healthy, but lived in a poor neighborhood and hade several dogs and cats at home. An infected dog or cat can shed up to one million Toxocara eggs each day, and these eggs can survive in the environment for many years. ${ }^{2,9}$ Our patient may have had geophagia, or might have eaten contaminated food.

Not only is CNS involvement in toxocariasis a rare clinical condition, but eosinophilic meningomyelitis is also rare in temperate countries, according to Dauriac-Le Masson et al. ${ }^{10}$ Interestingly, although our patient lives in a tropical country, he comes from a city in Southern Brazil, where the weather resembles that of temperate climates.

From the early 1950s through 2004, 28 cases of neurotoxocariasis were described, only 14 of which in children. ${ }^{4}$ In a survey of 47 cases of neurotoxocariasis, mean age at diagnosis was 22 years, and nearly $60 \%$ of patients were adults. 5

Toxocariasis has no racial predilection, and boys are usually more affected than girls, ${ }^{8}$ as in our report. The pathophysiology involves accidental infection of a human host, usually by ingestion of eggs from contaminated hands or food. There has been one report of a previously healthy 55-year-old woman with sudden-onset neurotoxocariasis after consumption of raw duck liver. ${ }^{9}$ Migration into the CNS is uncommon, and can provoke a more dramatic inflammatory response than the usual migratory path through the periphery. ${ }^{5}$

We presented a case with acute onset and involvement of both the central and peripheral nervous systems, as in a previously described case of meningomyelitis ${ }^{10}$; however, our patient also experienced concomitant cerebellar dysfunction. We are unaware of any previous reports describing this particular clinical association.

Concomitant central and peripheral neurologic involvement complicates an accurate topographic diagnosis. Other symptoms, such as cognitive dysfunction and urinary retention, can occur in neurotoxocariasis, as described in a 45 -year-old man, ${ }^{11}$ but these have not been reported in children.

In this case, cerebral involvement was clinically suggested by cognitive impairment, mental confusion, fluctuating level of consciousness, and lethargy, and was later confirmed by abnormal EEG activity with multifocal epileptiform discharges. EEG testing is of course nonspecific, but it is highly sensitive for cerebral dysfunction. Although we were unable to identify any structural brain abnormalities on neuroimaging studies, we cannot rule out cerebral dysfunction. We believe that clinical and electroencephalographic evidence of cerebral compromise are sufficient to confirm CNS involvement in this specific case. Thus, positive imaging findings should not be regarded as the gold standard for confirmation of cerebral neurotoxocariasis, at least in our case, particularly in light of the brief time elapsed between onset of symptoms and administration of anthelmintic treatment (less than five days).

Cerebellar involvement was made evident by unquestionable signs of cerebellar deficits, later confirmed by discrete cerebellar hypodensity on CT. 
Our patient had bladder and bowel incontinence that improved after anthelmintic treatment, confirming peripheral involvement. Peripheral nervous system involvement is rarely described in cases of neurotoxocariasis. These manifestations may go unrecognized, and are presumably underreported. ${ }^{3}$

Although epilepsy is a rare finding, it has been described in an 11-year-old girl with neurotoxocariasis, ${ }^{8}$ among a few other case reports. ${ }^{3}$ Our patient did not experience seizures, but his sleep EEG was strikingly abnormal, with frequent multifocal epileptiform discharges.

Differential diagnoses include cerebral glioma, ${ }^{2,5}$ brain abscess, ${ }^{8}$ acute disseminated encephalomyelitis, ${ }^{12}$ ando other helminthic or non-helminthic infections. ${ }^{5}$

There is a dearth of well-designed, controlled studies of neurotoxocariasis treatment, probably because of the rarity of the condition. Albendazole is one of the most commonly used drugs. ${ }^{3}$ Some authors claim that albendazole appears to be slightly more effective than thiabendazole, and is also better tolerated. ${ }^{5}$ However, other benzimidazoles can be used, such as mebendazole, flubendazole or thiabendazole, in a 3-to-4-week course of treatment.2,3 Our patient improved and became oligosymptomatic after a 5-week course of thiabendazole, the same drug used in other case reports. ${ }^{4,12}$ The usefulness of corticosteroids remains unclear. ${ }^{5}$

Prognosis depends on early diagnosis and proper treatment, in order to prevent secondary superinfection ${ }^{2}$, and can be very good in at least $60 \%$ of cases. ${ }^{5}$

In summary, neurotoxocariasis should be considered in the differential diagnosis of pediatric patients with unusual neurologic symptoms accompanied by CSF eosinophilia. Early diagnosis and proper treatment can prevent neurologic sequelae, especially in young patients. To our knowledge, this is the first report of a pediatric case of neurotoxocariasis with concomitant cerebral, cerebellar, and peripheral nervous system involvement in the international literature. No similar clinical presentation was found in a database search.

\section{Acknowlegments}

The authors are grateful to Dr. José Augusto Bragatti for his collaboration in the analysis of the electroencephalogram examinations.

\section{References}

1. Lowichik A, Ruff AJ. Parasitic infections of the central nervous system in children. Part II: Disseminated infections. J Child Neurol. 1995; 10:77-87.

2. Walker MD, Zunt JR. Neuroparasitic infections: nematodes. Semin Neurol. 2005;25:252-61.

3. Finsterer J, Auer $\mathrm{H}$. Neurotoxocarosis. Rev Inst Med Trop Sao Paulo.2007;49:279-87

4. Moreira-Silva SF, Rodrigues MG, Pimenta JL, Gomes CP, Freire LH, Pereira FE. Toxocariasis of the central nervous system: with report of two cases. Rev Soc Bras Med Trop. 2004;37:169-74.

5. Eberhardt O, Bialek R, Nägele T, Dichgans J. Eosinophilic meningomyelitis in toxocariasis: case report and review of the literature. Clin Neurol Neurosurg. 2005;107:432-8.

6. Mrissa R, Battikh R, Ben Abdelhafidh N, Jemli B, Azzouz O, Zaouali $\mathrm{J}$, et al. Toxocara canis encephalitis: case report. Rev Med Interne. 2005;26:829-32.

7. Moiyadi A, Mahadevan A, Anandh B, Shivashankar RS, Chickabasavaiah YT, Shankar SK. Visceral larva migrans presenting as multiple intracranial and intraspinal abscesses. Neuropathology. 2007;27:371-4

8. Bachli HJ, Minet JC, Gratzl O. Cerebral toxocariasis: a possible cause of epileptic seizure in children. Childs Nerv Syst. 2004;20:468-72.

9. Hoffmeister B, Glaeser S, Flick H, Pornschlegel S, Suttorp N, Bergmann F. Cerebral toxocariasis after consumption of raw duck liver. Am J Trop Med Hyg. 2007;76:600-2.

10. Dauriac-Le Masson V, Chochon F, DemeretS, Pierrot-Deseilligny C. Toxocara canis meningomyelitis. J Neurol. 2005;252:1267-8.

11. Scheid, R, Tina Jentzsch R, Schroeter ML. Cognitive dysfunction, urinary retention, and a lesion in the thalamus--beware of possible toxocariasis of the central nervous system. Clin Neurol Neurosurg. 2008;110:1054-7.

12. Marx C, Lin J, Masruha MR, Rodrigues MG, da Rocha AJ, Vilanova LC, et al. Toxocariasis of the CNS simulating acute disseminated encephalomyelitis. Neurology. 2007;69:806-7.

Correspondence:

Rudimar Riesgo

Av. Juca Batista, 8000/415

CEP 91780-000 - Porto Alegre, RS - Brazil

Tel.: + 55 (51) 3277.8415

Fax: +55 (51) 3333.1590

E-mail: rriesgo@hcpa.ufrgs.br 